\title{
Unimodal and crossmodal effects of endogenous attention to visual and auditory motion
}

\author{
ANTON L. BEER and BRIGITTE RÖDER \\ Philipps-University Marburg, Marburg, Germany
}

\begin{abstract}
Three experiments were conducted examining unimodal and crossmodal effects of attention to motion. Horizontally moving sounds and dot patterns were presented and participants' task was to discriminate their motion speed or whether they were presented with a brief gap. In Experiments 1 and 2 , stimuli of one modality and of one direction were presented with a higher probability $(p=.7)$ than other stimuli. Sounds and dot patterns moving in the expected direction were discriminated faster than stimuli moving in the unexpected direction. In Experiment 3, participants had to respond only to stimuli moving in one direction within the primary modality, but to all stimuli regardless of their direction within the rarer secondary modality. Stimuli of the secondary modality moving in the attended direction were discriminated faster than were oppositely moving stimuli. Results suggest that attending to the direction of motion affects perception within vision and audition, but also across modalities.
\end{abstract}

According to traditional textbooks, visual as well as auditory information initially is processed within modalityspecific cortical regions before it enters association cortex-such as parietal cortex - for multisensory integration. Different models have been proposed with regard to how top-down processes such as attention affect multisensory perception. Purely multimodal accounts (e.g., Farah, Wong, Monheit, \& Morrow, 1989) argue that attention operates at multisensory processing stages. Thus, attending to a particular feature - such as space-affects processing of visual, auditory, and tactile stimuli equally strongly. ${ }^{1}$ On the contrary, purely modality-specific accounts (e.g., Duncan, Martens, \& Ward, 1997; Robin \& Rizzo, 1989; Wickens, 1980) suggest that attention affects stimulus processing of the task-relevant modality only, possibly at processing stages at which the senses are still segregated. Recent research has provided evidence against both of these extreme views. For example, attention to the supramodal feature space appears to be neither purely modality specific nor purely multimodal. Our study was aimed to extend previous research on multisensory top-down processes by examining effects of endogenous attention to the direction of motion-another supramodal feature.

Spatial attention appears to operate at modalityspecific areas, but also across modalities. Spence and Driver (1996) asked participants to respond to auditory and visual stimuli appearing on either the left or right

Brigitte Röder is now at the Department of Biological Psychology and Neuropsychology, University of Hamburg. This study was funded by a grant (Ro 1226/4-1, 4-2, 4-3 to B.R.) from the Deutsche Forschungsgemeinschaft. Correspondence should be addressed to B. Röder, Department of Biological Psychology and Neuropsychology, University of Hamburg Von-Melle-Park 11, 20146 Hamburg, Germany (e-mail: brigitte.roeder@uni-hamburg.de or beer@staff.uni-marburg.de). side. Participants were instructed to expect sounds at a particular horizontal location. They were encouraged to do so since sounds were more likely to appear at one side than at the other side and sounds were more likely than lights. The participants' task was to judge the vertical position (top vs. bottom) of either sounds or lights, respectively. Spence and Driver found that auditory stimuli were discriminated faster when they appeared at the expected side rather than the unexpected side (unimodal attention effect). Moreover, lights presented at the location that was attended within audition were discriminated faster than lights appearing at the opposite side (crossmodal attention effect). Conversely, when space was attended within vision, lights as well as sounds appearing at the attended location were discriminated faster than stimuli at the unattended location. Since the participants had to judge the vertical position (top vs. bottom) of the stimuli while attention was manipulated along the horizontal axis (left vs. right) in Spence and Driver's experiments, the response dimension was independent from the cued spatial location. Facilitation effects, hence, most likely reflect perceptual enhancement rather than response priming. The findings reported by Spence and Driver argue against a purely modality-specific account since spatial attention within one modality facilitated spatial processing within another modality. However, facilitation within the unattended modality was weaker than within the attended modality, arguing against a purely multimodal account (e.g., Farah et al., 1989). Electrophysiological research further corroborates this notion (Eimer, 2001; Hillyard, Simpson, Woods, VanVoorhis, \& Münte, 1984; Teder-Sälejärvi, Münte, Sperlich, \& Hillyard, 1999). Attention to space was associated with changes of early modality-specific event-related potentials (ERPs) both for the attended and unattended modality. Again, crossmodal effects were smaller than unimodal effects. The 
ERP findings suggest that perceptual facilitation due to spatial attention takes place in sensory cortex, but operates across modalities (see also McDonald, Teder-Sälejärvi, Di Russo, \& Hillyard, 2003; McDonald, Teder-Sälejärvi, \& Hillyard, 2000). Besides the purely multimodal account, two other models have been proposed in order to account for crossmodal spatial attention effects. Spence and Driver (1996) proposed that attention operates within separable modality-specific systems that are, however, linked via lateral connections. By contrast, hierarchical (or hybrid) models of attention (e.g., Driver \& Spence, 2000; Posner, 1990) suggest that attention operates within unimodal and higher level multimodal stages of processing. Feedback projections from the multimodal system to unimodal systems are assumed in order to explain crossmodal effects occurring at modality-specific processing stages. $^{2}$

The present study examined whether unimodal effects of attention to motion features (such as direction of motion) are similar for vision and audition. Furthermore, we were interested in whether attention to motion affects processing of stimuli that move in the attended direction, but are of a different modality. There are reasons to assume similarities between spatial attention and attention to motion. Both motion features and space are supramodal - in contrast to modality-specific features such as color or timbre. Location as well as the speed and direction of motion can be encoded to some extent by all sensory systems (though negligible in degustation and olfaction). Moreover, both spatial and motion features are assumed to be processed within largely overlapping processing streams of the human brain. Spatial stimulus characteristics are thought to be processed within the dorsal visual (e.g., Ungerleider \& Haxby, 1994; Ungerleider \& Mishkin, 1982) or auditory (Maeder et al., 2001; Rauschecker \& Tian, 2000) pathway, respectively. Similarly, a dorsal visual and auditory pathway was proposed for the analysis of visual and auditory motion (e.g., Lewis, Beauchamp, \& DeYoe, 2000).

Until now, studies on the consequences of attention to motion have concentrated on visual motion perception. Visual stimuli moving in the attended direction are detected faster and more accurately (Sekuler \& Ball, 1977) and cause a stronger motion aftereffect (Alais \& Blake, 1999; Chaudhuri, 1990; von Grünau, Bertone, \& Pakneshan, 1998) than visual stimuli moving in the unattended direction. Moreover, attention to motion is associated with enhanced hemodynamic responses in motionsensitive brain regions (e.g., Beauchamp, Cox, \& DeYoe, 1997). Top-down modulation of neuronal activity was also observed in visual areas at a single-cell level in monkeys (Martínez-Trujillo \& Treue, 2002; Recanzone \& Wurtz, 2000; Treue \& Martínez-Trujillo, 1999). Finally, attended as opposed to unattended motion stimuli elicited a negative difference wave in ERP studies (AnlloVento \& Hillyard, 1996; Martín-Loeches, Hinojosa, \& Rubia, 1999). However, we have not found any reports showing similar attention effects within audition. Given that it has been shown that spatial attention affects processing of both visual and auditory stimuli (Spence \& Driver, 1996), a similar facilitation due to attention to motion may be expected within vision and audition. Hence, our first goal was to test whether endogenous attention to auditory motion also facilitates processing of auditory stimuli.

Furthermore, we expected crossmodal effects of attention to motion - similar to crossmodal effects of spatial attention (Spence \& Driver, 1996). In particular, when the direction of motion was attended within vision, processing of sounds moving in the same direction should be facilitated. Conversely, attention to auditory motion should reduce processing time for visual stimuli moving in the attended direction. A number of recent studies (reviewed by Soto-Faraco, Kingstone, \& Spence, 2003) have shown bottom-up, or exogenously driven, crossmodal interactions in motion processing.

In order to address these two questions, we conducted three experiments. In Experiments 1 and 2, a speed discrimination task and a gap discrimination task were used, respectively. Visual and auditory moving stimuli were presented randomly intermixed, and the participants were asked to respond to all stimuli. Attention was manipulated by increasing the probability of stimuli that were of the primary modality and moved in the attended direction. In Experiment 3, the same gap discrimination task was used as in Experiment 2, but attention was manipulated by asking the participants to respond only to stimuli moving in one direction within the primary modality. Within the secondary modality, responses were required to all stimuli irrespective of their motion direction.

\section{EXPERIMENT 1}

In Experiment 1, the participants had to judge the speed of horizontally moving dot patterns and sounds. They were instructed to respond to every stimulus, but to particularly attend to one motion direction and one modality, since these stimuli were presented with a higher probability than the remaining stimuli. We predicted that when vision was the primary modality, dot patterns moving in the expected direction would be processed faster than dot patterns moving in the opposite direction (unimodal attention effect). Similarly, we predicted that attending to the direction of auditory motion would facilitate speed discrimination of sounds moving in the attended direction (unimodal attention effect). Moreover, attention to motion within one modality was expected to reduce processing time for stimuli of the secondary modality moving in the attended rather than the unattended direction (crossmodal attention effects).

\section{Method}

Participants. Fifty-six undergraduate students voluntarily took part in this study as part of the course requirements for seeking an undergraduate degree in psychology. Data from 24 participants had to be excluded from further analysis because error rates were too high (see below) during the training or the test phase. Twelve of the 
remaining 32 participants were male and 20 were female. All but one were right-handed. All the participants reported normal hearing and normal or corrected-to-normal vision. Mean age was 24 years (range from 19 to 44 years). Written informed consent was obtained prior to the study.

Apparatus. The participants sat in front of a 19-in. screen (refresh rate of $75 \mathrm{~Hz}$, resolution of $800 \times 600$ pixels) and two speakers in a darkened room. The screen was positioned in the middle, and the two speakers were located $36^{\circ}$ to the left and right of the participants' midline (Figure 1). Both screen and speakers were vertically aligned at eye level. Viewing distance was about $30 \mathrm{~cm}$. The participant's head was immobilized by a chinrest.

Stimuli. Visual stimuli consisted of a random dot pattern (6,250 white pixels on a black background) that was presented on a black screen within a rectangle of about $24^{\circ}$ of visual angle (250 pixels) horizontally $\times 5^{\circ}(50$ pixels $)$ vertically. Twenty percent of the dots were moved coherently in a horizontal direction (either leftward or rightward); the remaining dots remained stationary. Motion was either slow $\left(7.5^{\circ} / \mathrm{sec}\right)$ or fast $(15 \% \mathrm{sec})$. All visual stimuli were presented for $293 \mathrm{msec}$ at the middle of the screen.

Auditory stimuli consisted of 300-msec white noise sounds. Sound pressure level was about $60 \mathrm{~dB}(\mathrm{~A})$ as measured at participants' ear position. Motion was simulated by continuously changing the interaural level differences (ILD) according to the law of sines (Bauer, 1961; Grantham, 1986). Consequently, a sound appeared to move horizontally between the two speakers along an annulus around the participant. Speed was chosen so that all auditory stimuli caused an above-threshold perception of motion. Moreover, the difference in speed was chosen to be above threshold on the basis of previous findings (Grantham, 1986). Accordingly, sounds moved with a speed of either $50 \% \mathrm{sec}$ (slow) or $120 \% \mathrm{sec}$ (fast). Moreover, sounds were located either to the right or left hemifield in order to eliminate spatial confounds. Such confounds may occur when participants attend to the start position of the sound rather than the direction of motion. Since sounds appeared at different positions, a spatial strategy was discouraged in the present study. Hence, rightward-moving sounds appeared to traverse a trajectory from $-36^{\circ}$ to $0^{\circ}$ or $0^{\circ}$ to $36^{\circ}$ (fast speed) and from $-12^{\circ}$ to $0^{\circ}$ or from $0^{\circ}$ to $12^{\circ}$ (slow speed). Start and end points for leftward-moving sounds were reversed.

Procedure. The participants' task was to decide whether a stimulus (visual or auditory) moved slowly or fast by briefly lifting either the index or middle finger of their dominant hand. Responses were detected with a light gate. Each trial started with the presentation of a stimulus (visual or auditory, moving either leftward or rightward, fast or slow). The trial-onset interval varied between

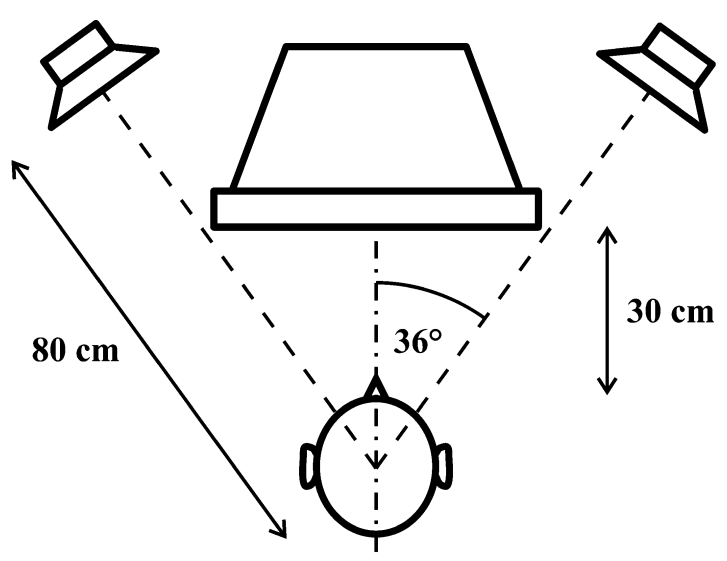

Figure 1. Schematic illustration of the experimental setup.
1,500 and 1,700 msec. Responses were recorded throughout each trial.

Prior to the experiment, speed discrimination was trained using three training blocks with a reduced amount of trials. During the first two training blocks, only visual or auditory stimuli, respectively, were presented. The third training block was composed of both visual and auditory stimuli. During training, feedback about the correctness of the response was provided after each trial. Each training block was repeated until a minimum of $80 \%$ correct responses was achieved. Eighteen participants failed to reach this criterion on the auditory training and a further 2 participants failed to succeed in the third training block. They were excluded from the experiment.

There were four experimental blocks with 240 trials each. Within each block, all types of stimuli were presented in random order. Prior to each block, participants were instructed to attend especially to a primary modality (vision or audition) and to a particular direction of motion (leftward or rightward) within that modality. This strategy was encouraged by varying the likelihood of visual and auditory, leftward- and rightward-moving stimuli. Hence, $70 \%$ of the stimuli were of the primary modality and moved in the expected direction, and $10 \%$ were of the primary modality but moved in the unexpected direction. Stimuli of the secondary modality $(20 \%)$ moved in either direction with equal probability. Half of the stimuli were slowly moving and half were fast moving. The order of the blocks as well as the response assignment of the fingers was counterbalanced across participants.

\section{Results}

Data from an additional 4 participants were excluded from further analysis because their overall error rates on auditory experimental trials exceeded $30 \%$. Response times less than $150 \mathrm{msec}$ or higher than $1,500 \mathrm{msec}$ were discarded. As suggested by Miller (1988), means rather than medians were used to calculate individual response times. Since we were interested in effects of attention to motion and in order to improve the reliability, all measures were pooled across both directions and speeds (note that leftward- and rightward-, slow- and fast-moving stimuli were equally likely).

Response times. Mean response times for visual and auditory stimuli and each condition of attended direction (attended vs. unattended) and priority (primary vs. secondary modality) are shown in Table 1 . A three-way within-subjects analysis of variance (ANOVA) encompassing the factors stimulus modality (visual vs. auditory), priority, and attended direction was conducted. Stimuli moving in the attended direction were discriminated faster than stimuli moving in the unattended direction, as reflected by a significant main effect of attended direction $[F(1,31)=47.9, p<.001]$. Furthermore, a significant main effect of stimulus modality $[F(1,31)=103.2, p<$ $.001]$ was found, indicating that responses to visual stimuli were faster than those to auditory stimuli. There was also a significant main effect of priority $[F(1,31)=236.1$, $p<.001]$, reflecting an intermodal attention effect; that is, responses to stimuli of the primary modality were faster than responses to stimuli of the secondary modality.

Moreover, there was a significant interaction between priority and attended direction $[F(1,31)=31.1, p<.001]$, indicating that the attention to motion effect was larger for unimodal conditions (primary modality) than for cross- 
Table 1

Mean Response Times (RTs) and Error Rates of Experiment 1

\begin{tabular}{|c|c|c|c|c|c|c|c|c|c|c|}
\hline \multirow[b]{3}{*}{ Modality } & \multicolumn{8}{|c|}{ Direction of Motion } & & \\
\hline & \multicolumn{4}{|c|}{ Attended } & \multicolumn{4}{|c|}{ Unattended } & \multicolumn{2}{|c|}{ Difference } \\
\hline & $\mathrm{RT}$ & $S D$ & Errors & $S D$ & RT & $S D$ & Errors & $S D$ & RT & Errors \\
\hline \multicolumn{11}{|l|}{ Primary } \\
\hline Visual stimuli & 620 & 69 & 8.9 & 6.2 & 651 & 78 & 11.1 & 7.2 & $31^{* * *}$ & $2.2^{*}$ \\
\hline Auditory stimuli & 724 & 76 & 15.1 & 8.6 & 757 & 75 & 15.6 & 8.4 & $33^{* * *}$ & .5 \\
\hline \multicolumn{11}{|l|}{ Secondary } \\
\hline Visual stimuli & 718 & 72 & 11.6 & 8.7 & 722 & 76 & 11.6 & 8.8 & 4 & .0 \\
\hline Auditory stimuli & 831 & 87 & 15.4 & 9.7 & 837 & 90 & 15.8 & 8.8 & 6 & .4 \\
\hline
\end{tabular}

Note-RTs are given in milliseconds. Error rates are expressed as percent. "Primary" means that the direction of motion was attended within the same modality (unimodal). "Secondary" means that the motion direction was attended in the other modality (crossmodal). The rightmost column depicts the attention to motion effect, which is the difference of RTs or error rates, respectively, to unattended minus attended stimuli. Significance levels were based on pairwise comparisons. They were familywise Bonferroni corrected. $n=32 .{ }^{*} p<.05 .{ }^{* * *} p<.001$.

modal conditions (secondary modality). Post hoc $t$ tests examining the attention to motion effect were conducted separately for each modality as well as uni- and crossmodal conditions, respectively. A Bonferroni correction was applied. Unimodal responses to stimuli moving in the attended direction were significantly faster than responses to stimuli moving in the unattended direction for both dot patterns $[t(31)=7.4, p<.001]$ and sounds $[t(31)=7.2$, $p<.001]$. These differences, however, were not significant when motion was attended within the other modality (crossmodal).

It might be argued that the faster responses to auditory stimuli moving in the attended direction reflect spatial attention rather than attention to motion (see above). For instance, the participants may have attended to the starting position of the sounds rather than to their direction of motion. Though this strategy was discouraged by our paradigm, we tested whether there was an attention to motion effect even when only response times to moving sounds starting in the middle location were included. There was almost no change of the unimodal attention effect. Sounds moving in the attended direction $(732 \mathrm{msec}$ ) were discriminated faster than sounds moving in the unattended direction $(764 \mathrm{msec})[t(31)=5.5, p<.001]$. Interestingly, the crossmodal effect on auditory stimuli now turned out to be marginally significant $[t(31)=2.32$, $p=.056]$. The participants responded faster to sounds that moved in a direction that was attended within vision $(781 \mathrm{msec})$ than to sounds moving in the opposite direction (801 msec).

Errors. A three-way within-subjects ANOVA on percentage of errors (see Table 1 ) revealed a significant main effect of stimulus modality $[F(1,31)=8.5, p=$ $.007]$, indicating that the participants made more errors in discriminating sounds $(15.5 \%)$ than in discriminating visual stimuli $(10.8 \%)$. No other main effects or interactions were significant. Nevertheless, pairwise $t$ tests (Bonferroni corrected) examining the attention to motion effect separately for each stimulus modality and priority condition (see right column of Table 1) revealed a significant unimodal effect for vision $[t(1,31)=2.8$, $p=.018]$. Responses to dot patterns were more accurate when they moved in a direction that was attended as opposed to unattended.

\section{Discussion}

The goal of Experiment 1 was to test whether sustained endogenous attention to the direction of motion facilitates processing of both visual and auditory stimuli and whether there is evidence for crossmodal processing gains. When the participants expected the direction in which a dot pattern or sound was moving, they were faster in discriminating its speed. Both visual and auditory facilitation were of similar size. Note that these attention effects may not be attributed to response competition, response priming, or a criterion shift, as discussed, for instance, by Santee and Egeth (1982) or Spence and Driver (1996, 1997). First, response competition and response priming would require that leftward- and rightwardmoving stimuli are linked to different responses. However, the kind of response ("slow" or "fast") was equally likely to occur regardless of whether the direction of motion was attended or not and regardless of which modality was more likely. Moreover, responses to stimuli moving in the attended direction were not only faster but also tended to be more accurate than responses to stimuli moving in the unattended direction. This argues against a speed-accuracy tradeoff (criterion shift).

It is also noteworthy that the feature that was analyzed faster was not the same as the feature to which attention was drawn. The participants did not selectively attend to the task-relevant feature speed, but to the direction of motion. Speed discrimination was chosen in Experiment 1 because it is closely related to motion direction. Experiment 2 tested whether attention to the direction of motion also affects the processing of features that are not or only remotely related to motion.

Experiment 1 revealed only subtle interactions between vision and audition mediated by motion. There was a marginally significant crossmodal attention to motion effect on sounds when only response times of sounds starting at the middle were entered into the analysis. 
Moreover, for the secondary modality, response time and error rate differences for stimuli moving in the attended versus unattended direction were always in the expected direction. However, these effects were small relative to crossmodal effects for spatial attention (Spence \& Driver, 1996). Two reasons may account for the lack of more reliable crossmodal effects. First, visual and auditory stimuli were not adequately spatially aligned. Whereas sounds appeared at two positions (left or right), dot patterns were always presented at the center of the screen. Spatial alignment, however, seems to be a modulating factor in order to observe crossmodal interactions between visual and auditory motion (Soto-Faraco, Lyons, Gazzaniga, Spence, \& Kingstone, 2002). Second, auditory motion stimuli were much harder to discriminate than visual motion stimuli, as reflected by the higher error rates and longer response times to sounds. Auditory speed differences were chosen to be above threshold on the basis of a previous report by Grantham (1986), but a relatively large proportion of participants were not able to sufficiently accomplish the auditory speed discrimination task. However, the participants in Grantham's study had experience in auditory motion experiments, whereas our participants were all novices - an important difference. Moreover, auditory motion thresholds appear to increase with adaptation (Grantham, 1992), which would explain why some of our participants were able to perform the training section, but failed to succeed in experimental blocks. In Experiment 2, we tried to reduce these potential problems. Auditory motion was made more salient, and a task was chosen that was easier to accomplish within audition. Additionally, spatial alignment between visual and auditory stimuli was improved.

\section{EXPERIMENT 2}

The goal of Experiment 2 was to test whether attention to motion facilitates perception of moving stimuli with regard to features that were not as closely related to motion as speed. The participants' task was to judge whether visual or auditory moving stimuli were presented continuously or briefly interrupted (gap discrimination). Furthermore, we hypothesized that crossmodal interactions are stronger when visual and auditory stimuli are more tightly spatially aligned. Hence, both visual and auditory stimuli were presented in either the left or right hemispace.

\section{Method}

Participants. Twenty-seven undergraduate students voluntarily took part in Experiment 2. They received credits that were required for their undergraduate degree in psychology. Data from 3 participants had to be excluded from further analyses because of too many errors during the training or the test phase. All but 3 of the remaining 24 participants ( 20 female, 4 male) were right-handed. All participants reported normal hearing and normal or corrected-to-normal vision. Mean age was 24 years (range from 20 to 37). Written informed consent was obtained prior to the study.

Apparatus. The apparatus was the same as in Experiment 1 except that the screen resolution was $640 \times 480$ pixels.

Stimuli. Visual stimuli consisted of a random dot pattern of 335 $\times 335$ pixels - half of them black and half white. All dots moved coherently within an area of $32^{\circ} \times 32^{\circ}$ of visual angle in a horizontal direction (either leftward or rightward). Motion speed was about $35 \%$ sec. In half of the trials, the dot pattern was presented continuously for $293 \mathrm{msec}$. In the other half, it disappeared from the screen for a brief period of time between 147 and $173 \mathrm{msec}$ (gap). Half of the visual stimuli were presented to the right of a central fixation cross and half were presented to the left in order to improve spatial alignment across modalities.

The auditory stimuli were similar to those used in Experiment 1. However, only fast-moving sounds $(120 \% \mathrm{sec})$ were used. As in the previous experiment, they appeared either in the left or in the right hemispace. Half of the sounds were briefly interrupted during presentation (silent gap between 148.5 and $151.5 \mathrm{msec}$ ), whereas the other half were presented continuously. Again, there were leftwardas well as rightward-moving sounds.

Procedure. The participants' task was to decide whether a stimulus (visual or auditory) was presented continuously or briefly interrupted by lifting either the index or middle finger of their dominant hand. The trial-onset interval varied from 1,500 to $1,700 \mathrm{msec}$.

Prior to the experiment, gap discrimination was trained using three training blocks with a reduced number of trials - similar to the training in Experiment 1 . A minimum of $80 \%$ correct responses was required for each training block. One participant failed to reach this criterion in the visual training condition.

There were four experimental blocks ( 240 trials). Within each block, all types of stimuli were presented in random order. Prior to a block, participants were instructed to attend especially to a primary modality (vision or audition) and to a particular motion direction within this modality. This strategy was encouraged by varying the likelihood of visual and auditory, leftward- and rightward-moving stimuli. As in the previous experiment, stimuli of the primary modality moving in the expected direction had a probability of $70 \%$, whereas all other modalities and directions had a likelihood of $10 \%$. Block order and response assignment were counterbalanced across participants. Feedback about the percentage of correct responses was provided after each block.

\section{Results}

Data from 2 participants had to be excluded from further analysis because their overall error rates on auditory experimental trials exceeded $30 \%$. Response times less than $150 \mathrm{msec}$ or higher than $1,500 \mathrm{msec}$ were discarded. Since we were mainly interested in effects of attention to motion, all measures were pooled across both locations, gap conditions, and directions (note that left and right, continuous and interrupted, leftward- and rightwardmoving stimuli were equally likely).

Response times. As in the previous experiment, a three-way within-subjects ANOVA (stimulus modality $\times$ priority $\times$ attended direction) was conducted. Attention to the direction of motion facilitated perception of stimuli moving in the attended direction, as reflected by a significant main effect of attended direction $[F(1,23)=8.4, p=$ $.008]$ and a significant interaction between priority and attended direction $[F(1,23)=6.9, p=.015]$. Separate $t$ tests (Bonferroni corrected) revealed that attention to motion facilitated responses of visual $[t(23)=3.5, p=.004]$ and auditory $[t(23)=4.3, p<.001]$ stimuli when they were of the primary modality (unimodal effects). Crossmodal differences between stimuli moving in the attended versus stimuli moving in the unattended direction were not significant.

Responses to moving dot patterns were faster than responses to moving sounds $[F(1,23)=4.8, p=.039]$. 
Moreover, stimuli that were of the primary modality were discriminated faster than stimuli of the secondary modality $[F(1,23)=269.3, p<.001]$. This intermodal attention effect was larger for sounds than for dot patterns, as reflected by a significant interaction of stimulus modality and priority $[F(1,23)=6.9, p=.015]$.

When only responses of sounds that started at the middle position were considered-in order to control for a potential spatial account - the same pattern of results emerged.

Errors. Error rates (Table 2) were submitted to the three-way within-subjects ANOVA (stimulus modality $\times$ priority $\times$ attended direction). No significant main effects or interactions were found.

\section{Discussion}

Experiment 2 addressed whether crossmodal interactions between auditory and visual attention to motion can be observed when the visual and auditory stimuli were equally discriminable and spatial alignment was improved. Though unimodal attention to motion effects (primary modality) were successfully replicated for both visual and auditory stimuli, no significant crossmodal attention to motion effects were detected. A third experiment was conducted in which we were striving to maximize the amount of attention devoted to the direction of motion.

\section{EXPERIMENT 3}

Experiment 3 was conducted in order to reveal whether top-down processes on motion operate across modalities. With regard to the primary modality, participants were asked to respond only to stimuli moving in the attended direction and to ignore stimuli moving in the opposite direction. However, they had to respond to all stimuli regardless of the direction of motion within the secondary modality.

\section{Method}

Participants. Nine volunteers took part in Experiment 3. They were compensated with 6 per hour or received credits. Data from
1 participant were excluded from further analysis because of too many errors on auditory trials. All of the remaining 8 participants (4 female, 4 male) were right-handed and reported normal hearing and normal or corrected-to-normal vision. Mean age was 23 years (range from 19 to 28 years). Written informed consent was obtained prior to the study.

Apparatus and Stimuli. The apparatus and stimuli were the same as in Experiment 2.

Procedure. There were four experimental blocks (240 trials each). Within each block, all types of stimuli were presented in random order. The participants' task (gap discrimination) was the same as in Experiment 2, but they were asked to respond only to stimuli moving in a particular direction within the primary modality. By contrast, responses were required to all stimuli (regardless of their direction of motion) within the secondary modality. Instructions were given prior to each block. In order to ensure that attention was predominantly focused on the primary modality, $80 \%$ of stimuli were of that modality. Leftward and rightward motion as well as left and right presentation were equally likely. Block order and response assignment were counterbalanced across participants. Feedback about the percentage of correct responses and false alarms was provided after each block.

Prior to the experiment, four training blocks were administered. They were similar to the experimental blocks, but a reduced number of trials were presented. Moreover, each response was directly followed by visually presented feedback. All participants were able to achieve the minimum criterion of $75 \%$ correct responses and less than $15 \%$ false alarms at each training block.

\section{Results}

Data from 1 participant were excluded from further analysis because of too many errors on auditory experimental trials $(>30 \%)$. Response times less than $150 \mathrm{msec}$ or higher than 1,500 msec were discarded.

Response times. Response times are depicted in Table 3. Only responses to stimuli of the secondary modality (crossmodal) were submitted to a two-way within-ANOVA encompassing the factors stimulus modality (visual vs. auditory) and attended direction (attended vs. unattended). A significant main effect of attended direction was found $[F(1,7)=33.2, p=.001]$, indicating that stimuli moving in a direction that was attended within the primary modality were discriminated faster than stimuli moving in the opposite direction. No significant differences were found between sounds and dot patterns.

Table 2

Mean Response Times (RTs) and Error Rates of Experiment 2

\begin{tabular}{|c|c|c|c|c|c|c|c|c|c|c|}
\hline \multirow[b]{3}{*}{ Modality } & \multicolumn{8}{|c|}{ Direction of Motion } & & \\
\hline & \multicolumn{4}{|c|}{ Attended } & \multicolumn{4}{|c|}{ Unattended } & \multicolumn{2}{|c|}{ Difference } \\
\hline & RT & $S D$ & Errors & $S D$ & RT & $S D$ & Errors & $S D$ & RT & Errors \\
\hline \multicolumn{11}{|l|}{ Primary } \\
\hline Visual stimuli & 631 & 45 & 6.5 & 5.2 & 648 & 49 & 7.1 & 8.4 & $17^{* *}$ & .6 \\
\hline Auditory stimuli & 626 & 50 & 6.0 & 4.6 & 643 & 53 & 6.2 & 7.0 & $17^{* * *}$ & .1 \\
\hline \multicolumn{11}{|l|}{ Secondary } \\
\hline Visual stimuli & 678 & 55 & 6.7 & 6.0 & 677 & 44 & 6.6 & 5.8 & -1 & -.1 \\
\hline Auditory stimuli & 714 & 78 & 6.5 & 5.3 & 720 & 84 & 6.4 & 4.5 & 6 & -.2 \\
\hline
\end{tabular}

Note-RTs are given in milliseconds. Error rates are expressed as percent. "Primary" means that the direction of motion was attended within the same modality (unimodal). "Secondary" means that the motion direction was attended in the other modality (crossmodal). The rightmost column depicts the attention to motion effect, which is the difference of response times or error rates, respectively, to unattended minus attended stimuli. Significance levels were based on pairwise comparisons. They were familywise Bonferroni corrected. $n=24 .{ }^{* *} p<.01 .{ }^{* * *} p<.001$. 
The crossmodal auditory attention to motion effect was significant even when only sounds starting in the middle were submitted to the analysis $[t(7)=5.5, p<$ $.001]$, arguing against a potential alternative spatial account (see above).

Errors. A two-way ANOVA on error rates for responses of the secondary modality (Table 3 ) revealed a significant interaction of stimulus modality and attended direction $[F(1,7)=6.9, p=.034]$. Visual stimuli moving in the attended direction were discriminated more accurately than visual stimuli moving in the unattended direction $[t(7)=$ $2.4, p=.100$ (Bonferroni corrected)]. No difference was found for sounds.

\section{Discussion}

The aim of Experiment 3 was to investigate crossmodal interactions of attention to motion between vision and audition. Though the direction of motion was task relevant only within the primary modality, responses to stimuli of the secondary modality were faster when they moved in the attended direction than when they moved in the unattended direction. Attention to auditory motion affected visual motion processing, and attention to visual motion affected auditory motion processing.

As for the unimodal attention to motion effects within Experiments 1 and 2, response priming may not explain these crossmodal effects since the response did not depend on the direction of motion. Furthermore, a speedaccuracy tradeoff (criterion shift) can be excluded. With regard to visual stimuli, participants responded both faster and more accurately to stimuli moving in the attended direction as compared with the other direction. No difference in error rates was found for sounds. Therefore, the crossmodal effects reported here most likely reflect facilitation on a perceptual level of processing.

It may be argued, however, that the crossmodal effects in the present experiment were due to response competition (Santee \& Egeth, 1982). Stimuli of the primary modality moving in the unattended direction might have been linked to a "no response" category, whereas stimuli of the secondary modality moving in that direction might have been linked to a "response" category. Such an account would not discredit crossmodal interactions in motion processing at a perceptual level since the prerequisite for response competition would be that the direction of motion was selected across modalities prior to the decision stage. Moreover, the paradigm used in Experiment 3 closely resembles the experimental design used in a recent ERP study examining crossmodal effects of spatial attention (Eimer \& Driver, 2000). In this study, participants had to respond to deviant stimuli at one location in the primary modality, but to all deviant stimuli of the secondary modality regardless of their location. ERPs of standard stimuli that did not require a response were enhanced as early as $100 \mathrm{msec}$ after stimulus onset when they were presented at the attended location, irrespective of whether they were of the primary or the secondary modality. Given that ERPs were analyzed only on standard stimuli, response competition is an unlikely explanation of the crossmodal effects of Eimer and Driver.

A somewhat puzzling result of Experiment 3 was that responses to sounds were slower when audition was the primary modality than when it was the secondary modality. At first glance, this finding seems to suggest that participants did not predominantly attend to the primary modality. However, if participants had indeed attended to the secondary rather than the primary modality, they would have attended to both rather than only one direction of motion. Thus, the direction of motion within the primary modality would have had no effect upon the secondary modality. Since such crossmodal effects were observed in Experiment 3, it is reasonable to conclude that participants sufficiently attended to the primary modality. Nevertheless, the question arises why there was essentially no intermodal attention effect-found in both Experiments 1 and 2-in Experiment 3. Note that in the third experiment, participants had to respond to all stimuli of the secondary modality, whereas they only had to respond to a subset of stimuli within the primary modality. Hence, the task within the primary modality required at least one processing step more than within the secondary modality - that is, deciding whether or not to re-

Table 3

Mean Response Times (RTs) and Error Rates of Experiment 3

\begin{tabular}{|c|c|c|c|c|c|c|c|c|c|c|}
\hline \multirow[b]{3}{*}{ Modality } & \multicolumn{8}{|c|}{ Direction of Motion } & & \\
\hline & \multicolumn{4}{|c|}{ Attended } & \multicolumn{4}{|c|}{ Unattended } & \multicolumn{2}{|c|}{ Difference } \\
\hline & RT & $S D$ & Errors & $S D$ & RT & $S D$ & Errors & $S D$ & RT & Errors \\
\hline \multicolumn{11}{|l|}{ Primary } \\
\hline Visual stimuli & 728 & 67 & 5.7 & 3.5 & - & & - & & - & - \\
\hline Auditory stimuli & 818 & 86 & 10.1 & 7.7 & - & & - & & - & - \\
\hline \multicolumn{11}{|l|}{ Secondary } \\
\hline Visual stimuli & 748 & 72 & 4.2 & 3.1 & 808 & 53 & 8.3 & 5.1 & $60^{* *}$ & $4.2^{\mathrm{a}}$ \\
\hline Auditory stimuli & 774 & 59 & 8.1 & 6.1 & 829 & 61 & 7.8 & 8.5 & $55^{* *}$ & -.3 \\
\hline \multicolumn{11}{|c|}{$\begin{array}{l}\text { Note-RTs are given in milliseconds. Error rates are expressed in percent. "Primary" denotes the } \\
\text { modality in which the direction of motion was attended. "Secondary" means that the motion direc- } \\
\text { tion was attended in the other modality (crossmodal). The rightmost column depicts the attention to } \\
\text { motion effect, which is the difference of response times or error rates, respectively, to unattended } \\
\text { minus attended stimuli. Significance levels were based on pairwise comparisons. They were family- } \\
\text { wise Bonferroni corrected. } n=8 . \quad \text { a } p<.10 .{ }^{* *} p<.01 \text {. }\end{array}$} \\
\hline
\end{tabular}


spond. This competition between responding and not responding presumably took some time and consumed all the time savings due to intermodal attention.

\section{GENERAL DISCUSSION}

\section{Unimodal Effects of Attention to Motion}

The present study tested whether sustained endogenous attention to the direction of motion facilitates processing of moving visual and auditory stimuli. Consistent with previous research (Alais \& Blake, 1999; Anllo-Vento \& Hillyard, 1996; Beauchamp et al., 1997; Chaudhuri, 1990; Martínez-Trujillo \& Treue, 2002; Martín-Loeches et al., 1999; Recanzone \& Wurtz, 2000; Sekuler \& Ball, 1977; Treue \& Martínez-Trujillo, 1999; von Grünau et al., 1998), our first two experiments demonstrated unimodal visual attention to motion effects. More specifically, when participants expected the direction a visual stimulus was moving, they were faster in discriminating its speed (Experiment 1) or a brief gap (Experiment 2).

Furthermore, Experiments 1 and 2 demonstrated for the first time that attention to the direction of auditory motion facilitated processing of sounds in much the same way that attention to visual motion facilitates visual perception. Sounds moving in the expected direction were discriminated faster than sounds moving in the unattended direction. Response time gains were almost the same for the unimodal auditory and visual conditions. Nevertheless, there was a small difference between vision and audition with regard to performance accuracy. Attention to visual motion tended to increase accuracy, whereas there was hardly any accuracy gain for auditory discrimination. A potential explanation could be that the visual modality is dominant in motion perception as it is in spatial perception (e.g., Welch \& Warren, 1980).

Attention to motion seems to operate within modalityspecific systems - at least to some extent. A purely multimodal account (e.g., Farah et al., 1989) of top-down influences on motion processing-even when feedback projections are assumed-would predict that unimodal and crossmodal effects are of similar size. ${ }^{3}$ In contrast to this prediction, the unimodal attention to motion effects in Experiments 1 and 2 were always larger than their crossmodal counterparts - which were hardly detectable. Converging evidence was provided by previous ERP studies showing that attending to the direction of visual motion affects brain activity at posterior, primarily visual, areas (Anllo-Vento \& Hillyard, 1996; Beauchamp et al., 1997; Martín-Loeches et al., 1999). Similarly, attending to the direction of moving sounds resulted in enhanced auditory ERPs at fronto-central scalp sites, suggesting an origin in modality-specific auditory brain areas (Beer \& Röder, 2004).

Unimodal attention to motion seems to operate at perceptual stages of processing. Decision or responserelated accounts such as response competition (Santee \& Egeth, 1982), criterion shifts, or response priming (see Spence \& Driver, 1996, 1997) may be rejected. In Ex- periments 1 and 2, the kind of response ("slow" or "fast," "continuous" or "interrupted") was independent of the direction of motion and modality. No speed-accuracy tradeoff was observed in any of the experiments. Since the requirements for the decision and/or response system did not vary across attention conditions, the response time facilitations in the present study appear to reflect processes prior to any decision stage and may be considered to be perceptual. ${ }^{4}$

It is noteworthy that performance gains due to attention may not necessarily result from enhanced processing of stimuli sharing the attended feature as, for instance, hypothesized by resource allocation models that are assuming a limited processing capacity (Luck, Hillyard, Mouloua, \& Hawkins, 1996). It may also be attributed to reduced processing of irrelevant stimuli (noise) that do not have the attended feature, as, for instance, assumed by uncertainty reduction models 5 (Shiu \& Pashler, 1994). Single-cell studies in monkeys (Martínez-Trujillo \& Treue, 2002; Treue \& Martínez-Trujillo, 1999) have revealed that motion-sensitive visual neurons not only show a response enhancement when the direction of motion has been attended, but also show a reduced response rate when the direction of motion has been ignored. Potentially, both signal enhancement as well as noise reduction may account for the unimodal attention effects observed in the present study.

\section{Crossmodal Effects of Attention to Motion}

The second goal of the present study was to test whether attending to the direction of motion within one modality affects processing of moving stimuli within another modality (crossmodal effects). In Experiments 1 and 2, only small and nonsignificant crossmodal effects could be observed, but very reliable crossmodal attention effects were detected in Experiment 3. Attending to the direction of motion for visual stimuli was associated with faster responses to sounds that moved in the taskrelevant direction. Conversely, auditory attention to motion speeded up responses to dot patterns moving in the attended direction. Note that participants had no strategic reason to attend to a particular direction of motion within the secondary modality since stimuli were equally likely to move toward the left or right. Instead, crossmodal effects occurred either because they were unavoidable or because it would have required extra effort to avoid them. Moreover, recent studies (Kitagawa \& Ichihara, 2002; Meyer \& Wuerger, 2001; Soto-Faraco et al., 2003; Soto-Faraco et al., 2002) have demonstrated crossmodal interactions between visual and auditory motion perception when participants were not explicitly asked to attend to a particular specification of motion features. For instance, the direction of motion had to be judged, but neither direction was endogenously attended. Hence, it may be assumed that the crossmodal interactions reported in these latter studies reflect automatic (exogenously driven) or maybe even "hard-wired" connections between visual and auditory motion processing. 
Crossmodal effects in the third experiment were very reliable, whereas they were almost absent in Experiments 1 and 2. Moreover, crossmodal effects in Experiment 3 seemed to be even larger than unimodal attention to motion effects of the first two experiments. At first glance, this appears to be counterintuitive. However, it has to be considered that a stronger allocation of attention may have been achieved in Experiment 3 than in the previous two, because participants could focus on one direction only in the primary modality. Nevertheless, it seems that attention to motion in one modality can be decoupled from other modalities. If so, what are the conditions under which crossmodal effects are to be observed? Being able to ignore a direction of motion may be one of them. In Experiment 3, the participants not only attended to one direction, but also actively ignored or inhibited the opposite direction within the primary modality. By contrast, inhibition was more difficult in Experiments 1 and 2 since responses were required to stimuli moving in either direction. As stated above, motion-sensitive neurons in monkeys not only show response enhancement but also response reduction due to attention to motion (MartínezTrujillo \& Treue, 2002; Treue \& Martínez-Trujillo, 1999). The large crossmodal effect in Experiment 3 could reflect crossmodal inhibition of stimuli moving in the ignored direction rather than only crossmodal enhancement of stimuli moving in the attended direction. Another prerequisite for crossmodal effects seems to be that both modalities must be task relevant. In a recent ERP study, we failed to find crossmodal effects using the same stimuli as those in the present Experiment 3 but not requiring responses for the secondary modality (Beer \& Röder, 2004). Finally, as discussed above, spatial alignment of stimuli across modalities appears to be important (Soto-Faraco et al., 2002).

Crossmodal effects in Experiment 3 discredit purely modality-specific accounts of attention to motion (e.g., Duncan et al., 1997; Robin \& Rizzo, 1989; Wickens, 1980). According to this view, attending to the direction of motion within one modality should not affect stimulus processing within another modality. Moreover, the similarities between visual and auditory unimodal attention to motion effects (equal in size) suggest that multimodal systems are involved as well. The models proposed in order to account for crossmodal spatial attention effects may also explain the present data. The separablebut-linked view (Spence \& Driver, 1996) assumes that attention operates within separable modality-specific systems, but these systems were linked via lateral connections. An implication of this notion would be that feature selection primarily takes place at early processing levels within modality-specific brain areas such as auditory and visual cortex, but these brain areas project to "unisensory" areas of other modalities. For instance, visual attention enhances processing within the visual cortex, but since there are connections from the visual to the auditory cortex, attention effects may also be observed within audition. Indeed, recent tracer studies in monkeys have demonstrated visual input to what was previously thought to be purely auditory cortex (Schroeder \& Foxe, 2002) and direct projections from auditory cortex to primary and secondary visual cortex (Falchier, Clavagnier, Barone, \& Kennedy, 2002; Rockland \& Ojima, 2003). Alternatively, hierarchical (or hybrid) models of attention (e.g., Driver \& Spence, 2000; Posner, 1990) suggest that attention operates within unimodal and higher-level multimodal stages of processing. Consistent with hierarchical models, McDonald et al. (2003) found that crossmodal effects of (exogenous) spatial attention first emerged in multisensory brain areas followed by activation in unisensory cortex.

\section{Conclusion}

In summary, the present study has demonstrated that endogenous attention modulates processing efficiency of both visual and auditory moving stimuli. Top-down processes appear to affect the analysis of motion stimuli on a perceptual and modality-specific level. Moreover, attention to visual motion affected processing of auditory motion, and attention to auditory motion modified processing of visual motion. This finding suggests that endogenously driven crossmodal interactions of feature processing along the dorsal pathway are not limited to spatial processing. Instead, the direction of motion - another supramodal feature - may play an important role in multisensory binding.

\section{REFERENCES}

Alais, D., \& Blake, R. (1999). Neural strength of visual attention gauged by motion adaptation. Nature Neuroscience, 2, 1015-1018.

Anllo-Vento, L., \& Hillyard, S. A. (1996). Selective attention to the color and direction of moving stimuli: Electrophysiological correlates of hierarchical feature selection. Perception \& Psychophysics, 58, 191-206.

BAUER, B. B. (1961). Phasor analysis of some stereophonic phenomena. Journal of the Acoustical Society of America, 33, 1536-1539.

Beauchamp, M. S., Cox, R. W., \& DeYoe, E. A. (1997). Graded effects of spatial and featural attention on human area MT and associated motion processing areas. Journal of Neurophysiology, 78, 516-520.

BeER, A. L., \& RöDER, B. (2004). Attention to motion enhances processing of both visual and auditory stimuli: An event-related potential study. Cognitive Brain Research, 18, 205-225.

Chaudhuri, A. (1990). Modulation of the motion aftereffect by selective attention. Nature, 344, 60-62.

Driver, J., \& SPENCE, C. (2000). Multisensory perception: Beyond modularity and convergence. Current Biology, 10, 731-735.

Duncan, J., Martens, S., \& WARD, R. (1997). Restricted attentional capacity within but not between sensory modalities. Nature, $\mathbf{3 8 7}$, 808-810.

EIMER, M. (2001). Crossmodal links in spatial attention between vision, audition, and touch: Evidence from event-related brain potentials. Neuropsychologia, 39, 1292-1303.

EIMER, M., \& DRIVER, J. (2000). An event-related brain potential study of cross-modal links in spatial attention between vision and touch. Psychophysiology, 37, 697-705.

Falchier, A., Clavagnier, S., Barone, P., \& Kennedy, H. (2002). Anatomical evidence of multimodal integration in primate striate cortex. Journal of Neuroscience, 22, 5749-5759.

Farah, M. J., Wong, A. B., Monheit, M. A., \& Morrow, L. A. (1989). Parietal lobe mechanisms of spatial attention: Modality-specific or supramodal? Neuropsychologia, 27, 461-470.

Grantham, D. W. (1986). Detection and discrimination of simulated 
motion of auditory targets in the horizontal plane. Journal of the Acoustical Society of America, 76, 1939-1949.

Grantham, D. W. (1992). Adaptation to auditory motion in the horizontal plane: Effect of prior exposure to motion on motion detectability. Perception \& Psychophysics, 52, 144-150.

Hillyard, S. A., Simpson, G. V., Woods, D. L., VanVoorhis, S., \& MÜNTE, T. F. (1984). Event-related brain potentials and selective attention to different modalities. In F. Reinoso-Suárez \& C. AjmoneMarsan (Eds.), Cortical integration (pp. 395-414). New York: Raven.

Kitagawa, N., \& Ichinara, S. (2002). Hearing visual motion in depth. Nature, 416, 172-174.

LABERGE, D. (2000). Networks of attention. In M. S. Gazzaniga (Ed.), The new cognitive neurosciences (2nd ed., pp. 711-724). Cambridge, MA: MIT Press.

Lewis, J. W., Beauchamp, M. S., \& DeYoe, E. A. (2000). A comparison of visual and auditory motion processing in human cerebral cortex. Cerebral Cortex, 10, 873-888.

Luck, S. J., Hillyard, S. A., Mouloua, M., \& Hawkins, H. L. (1996). Mechanisms of visual-spatial attention: Resource allocation or uncertainty reduction? Journal of Experimental Psychology: Human Perception \& Performance, 22, 725-737.

Maeder, P. P., Meuli, R. A., Adriani, M., Bellmann, A., Fornari, E., Thiran, J.-P., Pittet, A., \& Clarke, S. (2001). Distinct pathways involved in sound recognition and localization: A human fMRI study. NeuroImage, 14, 802-816.

Martínez-Trujilloo, J. C., \& Treue, S. (2002). Attentional modulation strength in cortical area MT depends on stimulus contrast. $\mathrm{Neu}$ ron, 35, 365-370.

MARTín-Loeches, M., Hinojosa, J. A., \& Rubia, F. J. (1999). Insights from event-related potentials into the temporal and hierarchical organization of the ventral and dorsal streams of the visual system in selective attention. Psychophysiology, 36, 721-736.

McDonald, J. J., Teder-Sälejärvi, W. A., Di Russo, F., \& Hillyard, S. A. (2003). Neural substrates of perceptual enhancement by crossmodal spatial attention. Journal of Cognitive Neuroscience, 15, 1019

McDonald, J. J., Teder-Sälejärvi, W. A., \& Hillyard, S. A. (2000). Involuntary orienting to sound improves visual perception. Nature, 407, 906-908.

MEYER, G. F., \& Wuerger, S. M. (2001). Cross-modal integration of auditory and visual motion signals. NeuroReport, 12, 2557-2560.

Miller, J. (1988). A warning about median reaction time. Journal of Experimental Psychology: Human Perception \& Performance, 14, 539-543.

MoOre, C. M., \& Egeth, H. (1998). How does feature-based attention affect visual processing? Journal of Experimental Psychology: Human Perception \& Performance, 24, 1296-1310.

Palmer, J., Ames, C. T., \& Lindsey, D. T. (1993). Measuring the effect of attention on simple visual search. Journal of Experimental Psychology: Human Perception \& Performance, 19, 108-130.

Posner, M. I. (1990). Hierarchical distributed networks in the neuropsychology of selective attention. In A. Caramazza (Ed.), Cognitive neuropsychology and neurolinguistics: Advances in models of cognitive function and impairment (pp. 187-210). Hillsdale, NJ: Erlbaum.

Rauschecker, J. P., \& Tian, B. (2000). Mechanisms and streams for processing of "what" and "where" in auditory cortex. Proceedings of the National Academy of Sciences, 97, 11800-11806.

RECANZONE, G. H., \& WURTZ, R. H. (2000). Effects of attention on MT and MST neuronal activity during pursuit initiation. Journal of Neurophysiology, 83, 777-790.

RoBIN, D. A., \& Rizzo, M. (1989). The effect of focal cerebral lesions on intramodal and cross-modal orienting of attention. In T. E. Prescott (Ed.), Clinical aphasiology (Vol. 18, pp. 61-74). Boston: College-Hill.

Rockland, K. S., \& OJima, H. (2003). Multisensory convergence in calcarine visual areas in macaque monkey. International Journal of Psychophysiology, 50, 19-26.

SANTEE, J. L., \& EgETH, H. E. (1982). Do reaction time and accuracy measure the aspects of letter recognition? Journal of Experimental Psychology: Human Perception \& Performance, 8, 489-501.
Schroeder, C. E., \& Foxe, J. J. (2002). The timing and laminar profile of converging inputs to multisensory areas of the macaque neocortex. Cognitive Brain Research, 14, 187-198.

SEKULER, R., \& BALL, K. (1977). Mental set alters visibility of moving targets. Science, 198, 60-62.

SHIU, L., \& PASHLER, H. (1994). Negligible effect of spatial precueing on identification of single digits. Journal of Experimental Psychology: Human Perception \& Performance, 20, 1037-1054.

Soto-Faraco, S., Kingstone, A., \& Spence, C. (2003). Multisensory contributions to the perception of motion. Neuropsychologia, 41, 1847-1862.

Soto-Faraco, S., Lyons, J., Gazzaniga, M., Spence, C., \& KingSTONE, A. (2002). The ventriloquist in motion: Illusory capture of dynamic information across sensory modalities. Cognitive Brain Research, 14, 139-146.

SPENCE, C., \& DrIVER, J. (1996). Audiovisual links in endogenous covert spatial attention. Journal of Experimental Psychology: Human Perception \& Performance, 22, 1005-1030.

SPENCE, C., \& DrIVER, J. (1997). On measuring selective attention to an expected sensory modality. Perception \& Psychophysics, 59, 389403.

Teder-Sälejärvi, W. A., Münte, T. F., Sperlich, F.-J., \& Hillyard, S. A. (1999). Intra-modal and cross-modal spatial attention to auditory and visual stimuli: An event-related brain potential study. $\underline{\operatorname{Cog}-}$ nitive Brain Research, 8, 327-343.

Treue, S., \& Martínez-Trujillo, J. C. (1999). Feature-based attention influences motion processing gain in macaque visual cortex. $\mathrm{Na}$ ture, 399, 575-579.

UNGERLEIDER, L. G., \& HAXBY, J. V. (1994). "What" and "where" in the human brain. Current Opinion in Neurobiology, 4, 157-165.

Ungerleider, L. G., \& MishKIn, M. (1982). Two cortical visual systems. In D. J. Ingle, M. A. Goodale, \& R. J. W. Mansfield (Eds.), Analysis of visual behavior (pp. 549-586). Cambridge, MA: MIT Press.

von Grünau, M. W., Bertone, A., \& Pakneshan, P. (1998). Attentional selection of motion states. Spatial Vision, 11, 329-347.

Welch, R. B., \& WARREN, D. H. (1980). Immediate perceptual response to intersensory discrepancy. Psychological Bulletin, 88, 638667.

WiCKENS, C. D. (1980). The structure of attentional resources. In R. S. Nickerson (Ed.), Attention and performance VIII (pp. 239-257). Hillsdale, NJ: Erlbaum.

\section{NOTES}

1. A purely multimodal account of attention would predict equally strong unimodal and crossmodal effects of attention even when feedback projections from multimodal to unimodal systems are assumed. These feedback projections would modify signal processing at unimodal areas irrespective of what modality is the primary modality in which the feature is attended. In order to explain the difference between unimodal and crossmodal effects, purely multimodal accounts would have to assume an additional low-level intermodal selection mechanism.

2. As discussed by LaBerge (2000), the site where attentional effects are observed is not necessarily the locus of the attentional control system. The controversy between the separable-but-linked view and the hierarchical models, therefore, is not about where crossmodal effects are to be observed, but about the origin of these effects. Whereas the separablebut-linked view assumes the locus within other modality-specific regions, hierarchical (or hybrid) models - at least when feedback projections are assumed - point to multimodal brain areas.

3. In order to explain strong unimodal but weak (or absent) crossmodal attention effects, purely multimodal accounts would need to assume an additional early intermodal selection mechanism by which only stimuli of the relevant (primary) modality are submitted to a motion processing stage. The intermodal attention effect found in Experiments 1 and 2 as well as a recent ERP finding showing that intermodal selection takes place prior to selection by motion (Beer \& Röder, 2004) would support this notion. However, all experiments of the present study required participants to respond to both modalities and, thus, intermodal selection never should have been complete. Despite this, 
hardly any crossmodal effects were observed in Experiments 1 and 2, making even this modified multimodal notion implausible.

4. Moore and Egeth (1998) have proposed that feature-based attention (e.g., to color or size) does involve a stimulus prioritization process instead of affecting the sensory quality of stimuli, as suggested for spatial attention. The present study does not allow us to distinguish between these two mechanisms for the case of attention to motion, but Anllo-Vento and Hillyard (1996) have found that selection of stimuli on the basis of motion direction does occur contingent on spatial selection: Attention to motion, hence, appears to operate at a later stage or higher level than spatial attention.
5. Uncertainty reduction models are sometimes labeled "decision" models (Palmer, Ames, \& Lindsey, 1993), implying that attentional processes are taking place at a postperceptual stage. However, an uncertainty reduction model assumes that unattended stimuli are "excluded, attenuated, or weighted less" (Shiu \& Pashler, 1994, p. 1039) or that stimuli are selected by “voluntary control” (Palmer et al., 1993, p. 116) prior to entering the decision stage. We consider these predecisional processes to be perceptual.

(Manuscript received October 1, 2003; revision accepted for publication March 29, 2004.) 